Charge-constrained auxiliary-density-matrix methods for the Hartree-Fock exchange contribution

Patrick Merlot, Róbert Izsák’ , Alex Borgoo, Thomas Kjærgaard, Trygve Helgaker, and Simen Reine’

Citation: The Journal of Chemical Physics 141, 094104 (2014); doi: 10.1063/1.4894267

View online: http://dx.doi.org/10.1063/1.4894267

View Table of Contents: http://aip.scitation.org/toc/jcp/141/9

Published by the American Institute of Physics

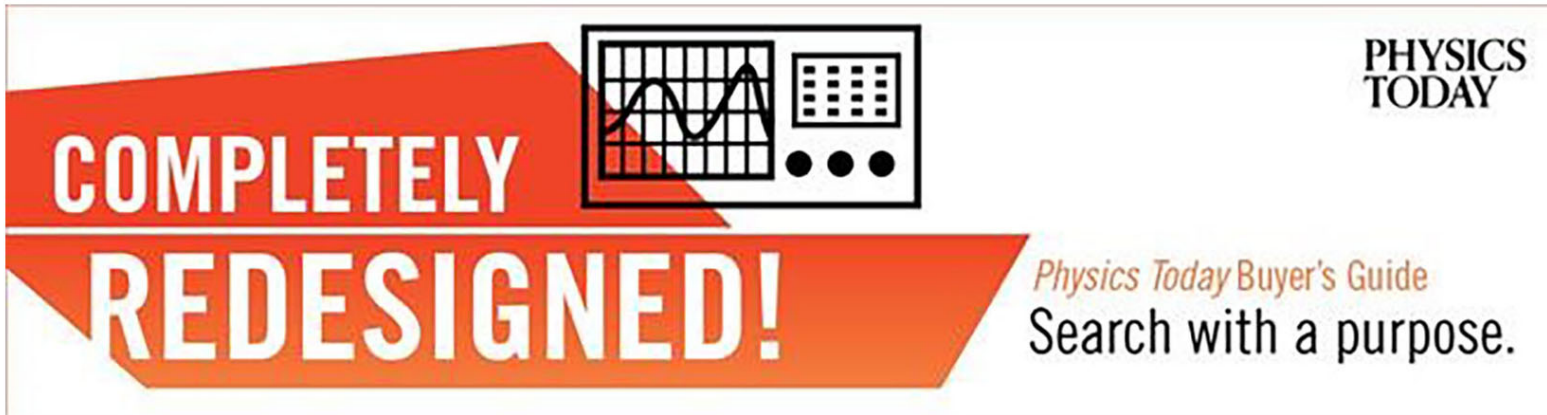




\title{
Charge-constrained auxiliary-density-matrix methods for the Hartree-Fock exchange contribution
}

\author{
Patrick Merlot, ${ }^{1}$ Róbert Izsák, ${ }^{1, a)}$ Alex Borgoo, ${ }^{1}$ Thomas Kjærgaard, ${ }^{2}$ Trygve Helgaker, ${ }^{1}$ \\ and Simen Reine ${ }^{1, b)}$ \\ ${ }^{1}$ Centre for Theoretical and Computational Chemistry, Department of Chemistry, University of Oslo, \\ P.O. Box 1033 Blindern, N-0315 Oslo, Norway \\ ${ }^{2}$ The qLEAP Center for Theoretical Chemistry, Department of Chemistry, Aarhus University, \\ 8000 Aarhus $C$, Denmark
}

(Received 5 June 2014; accepted 18 August 2014; published online 4 September 2014)

\begin{abstract}
Three new variants of the auxiliary-density-matrix method (ADMM) of Guidon, Hutter, and VandeVondele [J. Chem. Theory Comput. 6, 2348 (2010)] are presented with the common feature that they have a simplified constraint compared with the full orthonormality requirement of the earlier ADMM1 method. All ADMM variants are tested for accuracy and performance in all-electron B3LYP calculations with several commonly used basis sets. The effect of the choice of the exchange functional for the ADMM exchange-correction term is also investigated. (C) 2014 AIP Publishing LLC. [http://dx.doi.org/10.1063/1.4894267]
\end{abstract}

\section{INTRODUCTION}

The Hartree-Fock (HF) exchange term plays an important role in quantum chemistry. On the one hand, many of the available exchange-correction functionals of densityfunctional theory (DFT) contain some amount of HF exchange, which is computationally much more expensive than the Coulomb term. ${ }^{1}$ On the other hand, the HF method itself serves as a basis for most electron-correlation methods of wave-function theory (WFT). Furthermore, some of the more complex terms arising in post-HF WFT can be efficiently treated (after some reformulation) using algorithms developed for HF exchange evaluation. ${ }^{2,3}$ These facts indicate that the efficient evaluation of HF exchange is important for chemical systems of any size and at any desired level of accuracy within the framework of most methods available for practical use. In the present work, we propose an improved version of one of the methods available for efficient HF exchange evaluationnamely, the auxiliary-density-matrix method (ADMM) of Guidon, Hutter, and VandeVondele. ${ }^{4}$ Before discussing the details of our scheme, some of the exchange algorithms proposed in the literature are reviewed in this section.

In early HF implementations, the Coulomb and exchange terms were calculated simultaneously. The idea that the Coulomb and exchange terms are more efficiently computed separately ${ }^{5,6}$ than together in HF goes back to Almlöf's early observation that different integral batches are selected for the two terms as a result of prescreening. ${ }^{7,8}$ It is also worth pointing out that in DFT, the separate treatment of Coulomb and exchange contributions was from the beginning a natural strategy as shown by the early work of Slater on $X \alpha$ theory. ${ }^{9}$ Häser and Ahlrichs observed that, although the exchange term is potentially linear scaling, overall scaling is quadratic since the integral prescreening ${ }^{10}$ itself is quadratic with a small prefactor. For molecular systems, the sparsity

\footnotetext{
a)Electronic mail: roberiz@kjemi.uio.no

b)Electronic mail: s.s.reine@kjemi.uio.no
}

of the density can be exploited, giving rise to linear-scaling methods such as the order-N exchange method of Schwegler and Challacombe ${ }^{11}$ and the LinK algorithm of Ochsenfeld et al. ${ }^{12}$ For a recent review on linear-scaling methods, see Ref. 1.

A potentially powerful novel treatment of quantumchemical problems is what is called "multiresolution quantum chemistry," based on a multiresolution analysis in multiwavelet bases as an alternative computational framework, using the multiresolution adaptive numerical scientific simulation (MADNESS) software environment. ${ }^{13,14}$ The proposed treatment of the HF exchange is discussed in a separate article. $^{13}$

Due to the nature of the problem, it is clear that, if further approximations are introduced to obtain improved efficiency at a controlled amount of loss in accuracy, these should aim for the evaluation of integrals and/or the density. Among the most popular approximate integral-evaluation schemes is the resolution-of-identity (RI) or density-fitting (DF) scheme, which goes back to the work of Whitten ${ }^{15,16}$ and Baerends. ${ }^{17}$ Dunlap showed that fitting errors can be decreased by an order of magnitude if the fitting is carried out variationally in the Coulomb metric, ${ }^{18,19}$ which was later confirmed by Vahtras et al. ${ }^{20}$ in their study of various fitting metrics giving rise to an accurate DF scheme. Accurate approximate densities can also be obtained from fitting the Coulomb potential using Hermite Gaussian functions as in the auxiliary density functional theory (ADFT). ${ }^{21}$ It is also possible to obtain the equivalents of Dunlap's fitting equations ${ }^{19}$ within the ADFT framework, allowing the fitting coefficients to enter into the self-consistent-field (SCF) procedure. ${ }^{22}$ While the RI/DF approach has been successful for the Coulomb term, the RI exchange scheme goes back to the work of several groups, including Früchtl et al.,${ }^{23}$ Hamel et al. ${ }^{24}$ and Weigend. ${ }^{25}$ This scheme was later made linear scaling through the use of local orbital domains in the work of Polly et al. ${ }^{26}$ Another linearscaling exchange variant was proposed by Sodt et al. ${ }^{27}$ who 
applied their atomic RI scheme. In the work of Reine et al., ${ }^{28}$ the use of a local metric was combined with the robust fitting technique of Dunlap ${ }^{29}$ and formulated in a variational manner, yielding an accurate and potentially linear-scaling DF model. Accurate linear-scaling DF can also be achieved through the highly local pair-atomic $\mathrm{RI}^{30}$ and concentric-atomic $\mathrm{DF}^{31}$ methods. Note, however, that a potential problem with local DF methods is that the effective two-electron operator is not manifestly positive definite, which may lead to electron attraction rather than repulsion and variational collapse. ${ }^{30}$ Yet another way of achieving linear scaling is the application of the double asymptotic expansion of three centered integrals within a fitting scheme. ${ }^{32}$ The resulting method is free from variational problems.

Another method with a long history is the Choleskydecomposition technique, first applied to the two-electron repulsion problem by Beebe and Linderberg. ${ }^{33}$ In recent years, the Cholesky method has been revived and made into an efficient computational model. ${ }^{34-37}$ In the Local K method, ${ }^{35}$ strict error control is achieved by rigorous estimates of the orbital contributions to the exchange matrix, avoiding an ad hoc partitioning into local domains. The resulting method is up to two orders of magnitudes faster than the standard Cholesky SCF implementation.

The pseudospectral (PS) approach of Friesner ${ }^{38-40}$ originates in fluid dynamics and involves a transformation from quantities represented in physical space (function values) to their spectral-space representation (their expansion in some chosen basis). The idea is that the evaluation of integrals is cheaper in physical space: subsequently, the result is transformed back into spectral space. A detailed review of the method and its applications has been given by Martínez and Carter. ${ }^{41}$ The PS method itself may be regarded as a generalization of the discrete-variable-representation (DVR) method $^{42,43}$ of Light and co-workers and is related to a number of similar methods; see discussions in Refs. 41 and 44. Recently, the advantages of RI and PS methods were combined in the work of Yachmenev and Klopper. ${ }^{45}$

The chain-of-spheres (COS) approximation ${ }^{46,47}$ was introduced for the efficient evaluation of the Hartree-Fock exchange (hence the acronym COSX). COS is a seminumerical integration technique, where integration over one set of electron coordinates is carried out numerically. This can be done in an efficient manner at the cost of some loss in accuracy. The errors inherent to the numerical representation were later partially corrected for using the "overlap-fitting" procedure, ${ }^{47}$ which rescales basis-function values on a grid to yield the exact overlap matrix. The COS method is related to the PS method $^{47}$ but does not require the specific grid structure of the former. The relation of the COS method to (possibly robust) RI methods and the role of the complementary space in the treatment of numerical errors have recently been discussed in Ref. 48. An analogous semi-numerical method has been applied to two-component procedures and double-hybrid functionals by Plessow and Weigend. ${ }^{49}$

A recent series of papers ${ }^{50-52}$ deals with the tensorhypercontraction (THC) scheme, representing a fourth-order tensor (electron-repulsion integrals) by five second-order tensors, thereby reducing both formal scaling and storage requirements. ${ }^{50}$ In the least-squares variant of THC (LSTHC) scheme, ${ }^{51}$ four of the second-order tensors are chosen in a physically motivated manner, whereas the fifth is constrained to minimize the squared norm of the residual tensor.

All approximate methods discussed so far involve some alternative method of molecular-integral evaluation. The ADMM method works differently. ${ }^{4}$ Here, the exchange energy is split into two parts. One part consists of the exact HF exchange evaluated in a small auxiliary atomic basis set (from an auxiliary density matrix); the second part is a correction term, evaluated as the difference between the GGA (generalized gradient approximation) exchange in the full and auxiliary basis sets. This GGA exchange difference is assumed to be a good approximation to the corresponding exact exchange difference. The auxiliary density matrix can be obtained in the auxiliary basis in a number of ways, two of which are discussed by Guidon et al. ${ }^{4}$ The least-squares-deviation function between the orbitals obtained in the primary basis and in the auxiliary basis is minimized with respect to the auxiliary molecular orbital (MO) coefficients, with orthonormality constraints imposed on the auxiliary MOs (ADMM1) or without such constraints imposed (ADMM2). In this paper, we explore further variants of the ADMM scheme, with different constraints involving charges obtained with auxiliary MOs.

\section{THEORY}

\section{A. The ADMM approximation}

The expression for the ADMM exchange energy $(K)$ is based on the following trivial rearrangement of the total exchange energy:

$$
K(\mathbf{D})=k(\mathbf{d})+K(\mathbf{D})-k(\mathbf{d})
$$

where $\mathbf{D}$ is the density matrix in the primary atomic-orbital (AO) basis, while $\mathbf{d}$ is a density matrix obtained by projection of $\mathbf{D}$ to some (smaller) auxiliary AO basis. We here use upper-case letters to denote quantities evaluated in the primary basis, whereas lower-case letters refer to quantities in the auxiliary basis. The ADMM exchange energy $(\tilde{K})$ is obtained by replacing the exact-exchange terms $K(\mathbf{D})-k(\mathbf{d})$ in Eq. (1) with GGA-type exchange functionals $X(\mathbf{D})-x(\mathbf{d})$

$$
\begin{aligned}
\tilde{K}(\mathbf{D}) & =k(\mathbf{d})+X(\mathbf{D})-x(\mathbf{d}) \\
& =\sum_{\alpha \beta \gamma \delta} d_{\alpha \beta}(\alpha \gamma \mid \beta \delta) d_{\gamma \delta}+\int_{\mathbb{R}^{3}} \epsilon_{\mathrm{x}}[\rho] \mathrm{d} \mathbf{r}-\int_{\mathbb{R}^{3}} \epsilon_{\mathrm{x}}[\tilde{\rho}] \mathrm{d} \mathbf{r} .
\end{aligned}
$$

Here, $\epsilon_{\mathrm{x}}$ is the energy density of the GGA exchange functional used for the correction term, and the electron repulsion integrals $(\alpha \gamma \mid \beta \delta)$ are given in Mulliken (11|22) notation. Henceforth, we use indices $\mu, v, \ldots$ for the primary AOs and indices $i, j, \ldots$ for occupied MOs expanded in the primary AOs. For example, the density expanded in the primary basis $\rho$ in Eq. (2) is obtained as

$$
\rho=\sum_{i}^{\mathrm{occ}} \phi_{i}^{2}, \quad \phi_{i}=\sum_{\mu} C_{\mu i} \chi_{\mu} .
$$


For auxiliary AOs and MOs, we use indices $\alpha, \beta, \ldots \tilde{i}, \tilde{j}, \ldots$, respectively. The density expanded in the auxiliary basis $\tilde{\rho}$ in Eq. (2) then becomes

$$
\tilde{\rho}=\sum_{\tilde{i}}^{\mathrm{occ}} \phi_{\tilde{i}}^{2}, \quad \phi_{\tilde{i}}=\sum_{\alpha} c_{\alpha \tilde{i}} \chi_{\alpha} .
$$

Similar approximations using auxiliary densities for pure DFT exchange energy evaluation also exist. ${ }^{21}$

\section{B. The ADMM2 approximation}

In the ADMM2 approximation of Guidon et al., ${ }^{4}$ the projection is based on a least-squares fitting of the projected MOs, obtained by minimizing

$$
W_{2}=\sum_{i}^{\mathrm{occ}}\left\langle(i-\tilde{i})^{2}\right\rangle,
$$

where we have introduced the compact notation

$$
\left\langle(i-\tilde{i})^{2}\right\rangle=\int\left(\phi_{i}(\mathbf{r})-\phi_{\tilde{i}}(\mathbf{r})\right)^{2} \mathrm{~d} \mathbf{r}
$$

with respect to the projected MO coefficients $c_{\alpha i}$. Expansion of the expectation value gives

$W_{2}=\sum_{i}\left(\left\langle i^{2}\right\rangle-2\langle i \tilde{i}\rangle+\left\langle\tilde{i}^{2}\right\rangle\right)=\operatorname{Tr}\left(\mathbf{C}^{\mathrm{T}} \mathbf{S C}-2 \mathbf{c}^{\mathrm{T}} \mathbf{Q C}+\mathbf{c}^{\mathrm{T}} \mathbf{s c}\right)$,

where $\mathbf{c}$ and $\mathbf{C}$ are matrices containing the $\mathrm{MO}$ coefficients in the auxiliary and primary AO bases, respectively; $\mathbf{S}$ is the AO overlap matrix in the primary basis with elements $S_{\mu \nu}$ $=\langle\mu \nu\rangle ; \mathbf{s}$ is the $\mathrm{AO}$ overlap matrix in the auxiliary basis with elements $s_{\alpha \beta}=\langle\alpha \beta\rangle$; and $\mathbf{Q}$ is the mixed auxiliary-primary AO overlap matrix with elements $Q_{\alpha \mu}=\langle\alpha \mu\rangle$. Differentiating Eq. (7) with respect to $\mathbf{c}$ and setting the result equal to zero, we obtain the following linear sets of equations:

$$
\mathbf{c}_{2}=\mathbf{s}^{-1} \mathbf{Q C}=\mathbf{T C}, \quad \mathbf{T} \equiv \mathbf{s}^{-1} \mathbf{Q},
$$

where the subscript " 2 " indicates that the coefficients are those of the ADMM2 model.

Having determined the expansion coefficients $\mathbf{c}_{2}$, the projected density matrix $\mathbf{d}_{2}$ can be written in terms of the regular AO density matrix $\mathbf{D}$ as

$$
\mathbf{d}_{2}=\mathbf{T C C}^{\mathrm{T}} \mathbf{T}^{\mathrm{T}}=\mathbf{T D T}^{\mathrm{T}} .
$$

We now obtain the following expression for the ADMM2 exchange matrix $\tilde{K}_{\mu \nu}=\partial \tilde{K} / \partial D_{\mu \nu}$ :

$$
\tilde{\mathbf{K}}_{2}=\mathbf{X}(\mathbf{D})+\mathbf{T}^{\mathrm{T}}\left(\mathbf{k}\left(\mathbf{d}_{2}\right)-\mathbf{x}\left(\mathbf{d}_{2}\right)\right) \mathbf{T},
$$

where

$$
\begin{gathered}
X_{\mu \nu}(\mathbf{D})=\int_{\mathbb{R}^{3}} v_{\mathrm{x}}[\rho](\mathbf{r}) \chi_{\mu}(\mathbf{r}) \chi_{\nu}(\mathbf{r}) \mathrm{d} \mathbf{r}, \\
k_{\alpha \beta}(\mathbf{d})=\sum_{\gamma \delta}(\alpha \gamma \mid \beta \delta) d_{\gamma \delta}, \\
x_{\alpha \beta}(\mathbf{d})=\int_{\mathbb{R}^{3}} v_{\mathrm{x}}[\tilde{\rho}](\mathbf{r}) \chi_{\alpha}(\mathbf{r}) \chi_{\beta}(\mathbf{r}) \mathrm{d} \mathbf{r},
\end{gathered}
$$

where $v_{\mathrm{x}}[\rho](\mathbf{r})=\delta X / \delta \rho(\mathbf{r})$ is the exchange potential.

\section{The ADMM1 approximation}

In the alternative ADMM1 formulation, the orthonormality of the projected MOs is enforced by a standard Lagrangian formalism, introducing the Lagrange multipliers $\lambda_{i j}$

$$
W_{1}=\sum_{i}^{\mathrm{occ}}\left\langle(i-\tilde{i})^{2}\right\rangle+\sum_{i j}^{\mathrm{occ}} \lambda_{i j}\langle i j-\tilde{i} \tilde{j}\rangle .
$$

Proceeding in the same manner as for the ADMM2 Lagrangian, we obtain the following coefficients of the auxiliary MOs:

$$
\mathbf{c}_{1}=\mathbf{c}_{2} \mathbf{P}^{-1 / 2}, \quad \mathbf{P}=\mathbf{c}_{2}^{\mathrm{T}} \mathbf{s c}_{2} .
$$

Hence, the ADMM1 MOs are simply the symmetrically orthonormalized ADMM2 orbitals. This is what one expects, since Löwdin's symmetric orthonormalization scheme ${ }^{53}$ was shown to yield orbitals closest to the original ones in a least square sense by Carlson and Keller, ${ }^{54}$ and later in a more transparent form by Mayer. ${ }^{55}$ From $\mathbf{c}_{1}$, we obtain the orthonormality-constrained ADMM1 projected density matrix

$$
\mathbf{d}_{1}=\mathbf{T C} \mathbf{P}^{-1} \mathbf{C}^{\mathrm{T}} \mathbf{T}^{\mathrm{T}} .
$$

Unlike the unconstrained ADMM2 density matrix in Eq. (9), the orthonormality-constrained auxiliary density $\mathbf{d}_{1}$ matrix cannot be expressed directly in terms of the regular $\mathrm{AO}$ density matrix $\mathbf{D}$. In the ADMM1 formulation, therefore, the construction of a proper KS matrix $\tilde{K}_{\mu \nu}=\partial \tilde{K} / \partial D_{\mu \nu}$ is more complicated.

\section{The ADMMQ approximation}

In view of the difficulties associated with the orthonormality-constrained ADMM1 approximation, we propose a simpler charge-constrained ADMM formulation, denoted ADMMQ. The ADMMQ formulation is based on the following Lagrangian:

$$
W_{\mathrm{Q}}=\sum_{i}^{\mathrm{occ}}\left\langle(i-\tilde{i})^{2}\right\rangle+\lambda\left(\frac{N}{2}-\sum_{i}^{\mathrm{occ}}\left\langle\tilde{i}^{2}\right\rangle\right),
$$

where the multiplier $\lambda$ is to be adjusted so that the resulting auxiliary density matrix $\mathbf{d}_{\mathrm{Q}}$ satisfies the following condition:

$$
2 \operatorname{Tr}\left(\mathbf{d}_{\mathrm{Q}} \mathbf{s}\right)=N, \quad \mathbf{d}_{\mathrm{Q}}=\mathbf{c}_{\mathrm{Q}} \mathbf{c}_{\mathrm{Q}}^{\mathrm{T}},
$$

where $N$ is the number of electrons. The factor 2 arises due to the fact that the occupied orbitals are normalized to 1 , and the double occupancy is not taken care of in our definition of the density. Note that the ADMMQ Lagrangian $W_{\mathrm{Q}}$ in Eq. (17) is obtained from the ADMM1 Lagrangian $W_{1}$ in Eq. (14) by setting

$$
\lambda_{i j}=\lambda \delta_{i j}
$$

where $\delta_{i j}$ is the Kronecker delta, meaning that we ignore the orthogonality conditions and replace the normalization conditions on the individual MOs by an overall normalization condition, setting the overall "charge" equal to $N$. 
Minimization of the Lagrangian in Eq. (17) leads to a simple rescaling of the transformation matrix in Eq. (8),

$$
\mathbf{c}_{\mathrm{Q}}=\xi^{1 / 2} \mathbf{s}^{-1} \mathbf{Q C}=\xi^{1 / 2} \mathbf{c}_{2}, \quad \xi=(1-\lambda)^{-2},
$$

and the following auxiliary density matrix:

$$
\mathbf{d}_{\mathrm{Q}}=\xi \mathbf{d}_{2}=\mathbf{T}_{\mathrm{Q}} \mathbf{D} \mathbf{T}_{\mathrm{Q}}^{\mathrm{T}}, \quad \mathbf{T}_{\mathrm{Q}}=\xi^{1 / 2} \mathbf{T} .
$$

From the constraint $2 \operatorname{Tr} \mathbf{d}_{\mathrm{Q}} \mathbf{s}=N$, we obtain

$$
\xi=\frac{N}{N_{2}}=\frac{\operatorname{Tr}(\mathbf{D S})}{\operatorname{Tr}\left(\mathbf{d}_{2} \mathbf{s}\right)},
$$

where the scaling factor $\xi$ is given here as the ratio of the number of particles $N$ in $\mathbf{D}$ and $N_{2}$ in $\mathbf{d}_{2}$. Comparing the symmetry, normalization, and idempotency conditions of the auxiliary density matrices in the ADMM1, ADMM2, and ADMMQ schemes, we find

$$
\begin{gathered}
\mathbf{d}_{1}^{\mathrm{T}}=\mathbf{d}_{1}, \quad 2 \operatorname{Tr}\left(\mathbf{d}_{1} \mathbf{s}\right)=N, \quad \mathbf{d}_{1} \mathbf{s d}_{1}=\mathbf{d}_{1}, \\
\mathbf{d}_{2}^{\mathrm{T}}=\mathbf{d}_{2}, \quad 2 \operatorname{Tr}\left(\mathbf{d}_{2} \mathbf{s}\right) \neq N, \quad \mathbf{d}_{2} \mathbf{s d}_{2} \neq \mathbf{d}_{2}, \\
\mathbf{d}_{\mathrm{Q}}^{\mathrm{T}}=\mathbf{d}_{\mathrm{Q}}, \quad 2 \operatorname{Tr}\left(\mathbf{d}_{\mathrm{Q}} \mathbf{s}\right)=N, \quad \mathbf{d}_{\mathrm{Q}} \mathbf{s d}_{\mathrm{Q}} \neq \mathbf{d}_{\mathrm{Q}} .
\end{gathered}
$$

By dropping the idempotency condition and keeping the normalization condition, the ADMMQ density matrix has the correct charge (as in the ADMM1 scheme) and a well-defined $\mathrm{KS}$ exchange matrix (as in the ADMM2 schemes). It would be possible to apply McWeeny purification to the ADMMQ matrix ${ }^{56}$ but this would change the normalization of the matrix and also destroy the simple relationship between $\mathbf{d}_{\mathrm{Q}}$ and D in Eq. (21).

To enforce the constraints throughout the SCF procedure and to simplify evaluation of energy derivatives, Eq. (2) can be modified into the following Lagrangian (when replacing the regular exchange energy in the total KS energy expression):

$$
\tilde{K}_{\mathrm{Q}}=X(\mathbf{D})+k\left(\mathbf{d}_{\mathrm{Q}}\right)-x\left(\mathbf{d}_{\mathrm{Q}}\right)+2 \Lambda\left[\operatorname{Tr}(\mathbf{D S})-\operatorname{Tr}\left(\mathbf{d}_{\mathrm{Q}} \mathbf{s}\right)\right],
$$

with the corresponding ADMMQ exchange matrix (obtained as the derivative with respect to the primary density matrix D)

$$
\tilde{\mathbf{K}}_{\mathrm{Q}}=\mathbf{X}(\mathbf{D})+\mathbf{T}_{\mathrm{Q}}^{\mathrm{T}}\left(\mathbf{k}\left(\mathbf{d}_{\mathrm{Q}}\right)-\mathbf{x}\left(\mathbf{d}_{\mathrm{Q}}\right)\right) \mathbf{T}_{\mathrm{Q}}+\Lambda\left(\mathbf{S}-\mathbf{T}_{\mathrm{Q}}^{\mathrm{T}} \mathbf{s} \mathbf{T}_{\mathrm{Q}}\right) .
$$

Note that the charge-constraint term in Eq. (26) does not contribute to the energy itself since $2 \operatorname{Tr}(\mathbf{D S})=2 \operatorname{Tr}\left(\mathbf{d}_{\mathrm{Q}} \mathbf{s}\right)=N$ by construction. There is no factor 2 for the constraint in Eq. (27), since there is no need for double counting the orbitals in the exchange matrix as opposed to the exchange energy in Eq. (26). The Lagrange multiplier $\Lambda$ is fixed by requiring that the derivative of Eq. (26) with respect to $\lambda$ should vanish, yielding

$$
\Lambda=\frac{2}{N} \operatorname{Tr}\left(\left(\mathbf{k}\left(\mathbf{d}_{\mathrm{Q}}\right)-\mathbf{x}\left(\mathbf{d}_{\mathrm{Q}}\right)\right) \mathbf{d}_{\mathrm{Q}}\right) .
$$

\section{E. The ADMMS and ADMMP approximations}

The auxiliary ADMMQ density matrix $\mathbf{d}_{\mathrm{Q}}$ is related to the auxiliary ADMM2 density matrix $\mathbf{d}_{2}$ by a simple scaling, $\mathbf{d}_{\mathrm{Q}}=\xi \mathbf{d}_{2}$, as in Eq. (21), where the scaling factor is the one defined in Eq. (22). Such a scaling of $\mathbf{d}_{2}$ leads to the following scaling of the exact exchange and functional exchange:

$$
k\left(\mathbf{d}_{\mathrm{Q}}\right)=\xi^{2} k\left(\mathbf{d}_{2}\right),
$$

$$
x_{\mathrm{LDA}}\left(\mathbf{d}_{\mathrm{Q}}\right)=\xi^{4 / 3} x_{\mathrm{LDA}}\left(\mathbf{d}_{2}\right) \text {. }
$$

In non-LDA cases, the $\xi$ dependence may be different although we may take the leading term to be the same. The effective scaling of various functionals has been investigated in the literature. ${ }^{57,58}$ As a result, the correction term depends in a complicated manner on $\xi$ and can be approximated by its leading term approximation

$$
k\left(\mathbf{d}_{\mathrm{Q}}\right)-x\left(\mathbf{d}_{\mathrm{Q}}\right) \approx \xi^{2} k\left(\mathbf{d}_{2}\right)-\xi^{4 / 3} x\left(\mathbf{d}_{2}\right)
$$

In the ADMMQ method, the different scaling of the exact and DFT exchange functionals with respect to $\xi$ means that the energy may be variationally lowered during the SCF optimization by reducing $N_{2}$ and increasing $\xi$, thus possibly resulting in a large deviation of the converged total energy relative to the ADMM2 energy.

In the ADMMS scheme, the different scaling of $k$ and $x$ is avoided by multiplying the $x\left(\mathbf{d}_{\mathrm{Q}}\right)$ term in Eq. (26) by a factor $\xi^{2 / 3}$ and evaluating the functional using the scaled projected density as in ADMMQ, yielding

$$
\tilde{K}_{\mathrm{S}}=X(\mathbf{D})+k\left(\mathbf{d}_{\mathrm{Q}}\right)-\xi^{2 / 3} x\left(\mathbf{d}_{\mathrm{Q}}\right)+2 \Lambda\left[\operatorname{Tr}(\mathbf{D S})-\operatorname{Tr}\left(\mathbf{d}_{\mathrm{Q}} \mathbf{s}\right)\right] .
$$

In the ADMMP scheme, the scaling problem of $k$ and $x$ is solved by assuming that the LDA scaling in Eq. (30) holds explicitly for all functionals, thus we may factorize with respect to $\xi$, and use the functionals with the unscaled projected density, yielding

$$
\tilde{K}_{\mathrm{P}}=X(\mathbf{D})+\xi^{2}\left[k\left(\mathbf{d}_{2}\right)-x\left(\mathbf{d}_{2}\right)\right]+2 \Lambda\left[\operatorname{Tr}(\mathbf{D S})-\xi \operatorname{Tr}\left(\mathbf{d}_{2} \mathbf{s}\right)\right] .
$$

Since both exact and functional exchange now depend quadratically on $\xi$, variational lowering and large errors are avoided.

In the ADMMP scheme, $\Lambda$ is straightforwardly evaluated as

$$
\Lambda=\frac{2 \xi^{2}}{N}\left[k\left(\mathbf{d}_{2}\right)-x\left(\mathbf{d}_{2}\right)\right]
$$

and the ADMMP exchange matrix is defined as

$$
\tilde{\mathbf{K}}_{\mathrm{P}}=\mathbf{X}(\mathbf{D})+\xi^{2} \mathbf{T}^{\mathrm{T}}\left[\mathbf{k}\left(\mathbf{d}_{2}\right)-\mathbf{x}\left(\mathbf{d}_{2}\right)\right] \mathbf{T}+\Lambda\left(\mathbf{S}-\xi \mathbf{T}^{\mathrm{T}} \mathbf{s} \mathbf{T}\right) .
$$

In the ADMMS scheme, the $\Lambda$ expression is somewhat more complicated

$$
\Lambda=\frac{2}{N}\left[\operatorname{Tr}\left(\mathbf{k}\left(\mathbf{d}_{\mathrm{Q}}\right) \mathbf{d}_{\mathrm{Q}}\right)-\xi^{\frac{2}{3}}\left(\frac{1}{3} x\left(\mathbf{d}_{\mathrm{Q}}\right)+\operatorname{Tr}\left(\mathbf{x}\left(\mathbf{d}_{\mathrm{Q}}\right) \mathbf{d}_{\mathrm{Q}}\right)\right)\right],
$$


TABLE I. Terms in the ADMM energy Lagrangian $\tilde{K}=X(\mathbf{D})+\Delta k+2 \Lambda \Delta N$.

\begin{tabular}{lccccc}
\hline \hline Terms & ADMM1 & ADMM2 & ADMMQ & ADMMS & ADMMP \\
\hline$\Delta k$ & $k\left(\mathbf{d}_{1}\right)-x\left(\mathbf{d}_{1}\right)$ & $k\left(\mathbf{d}_{2}\right)-x\left(\mathbf{d}_{2}\right)$ & $k\left(\mathbf{d}_{\mathrm{Q}}\right)-x\left(\mathbf{d}_{\mathrm{Q}}\right)$ & $k\left(\mathbf{d}_{\mathrm{Q}}\right)-\xi^{2 / 3} x\left(\mathbf{d}_{\mathrm{Q}}\right)$ & $\xi^{2}\left(k\left(\mathbf{d}_{2}\right)-x\left(\mathbf{d}_{2}\right)\right)$ \\
$\Delta N$ & $\ldots$ & $\ldots$ & $\operatorname{Tr}(\mathbf{D S})-\operatorname{Tr}\left(\mathbf{d}_{\mathrm{Q}} \mathbf{s}\right)$ & $\operatorname{Tr}(\mathbf{D S})-\operatorname{Tr}\left(\mathbf{d}_{\mathrm{Q}} \mathbf{s}\right)$ & $\operatorname{Tr}(\mathbf{D S})-\operatorname{Tr}\left(\mathbf{d}_{\mathrm{Q}} \mathbf{s}\right)$ \\
$\Lambda \times \frac{N}{2}$ & $\ldots$ & $\ldots$ & $\left.\operatorname{Tr}\left(\mathbf{k}\left(\mathbf{d}_{\mathrm{Q}}\right)-\mathbf{x}\left(\mathbf{d}_{\mathrm{Q}}\right)\right) \mathbf{d}_{\mathrm{Q}}\right)$ & $\operatorname{Tr}\left(\mathbf{k}\left(\mathbf{d}_{\mathrm{Q}}\right) \mathbf{d}_{\mathrm{Q}}\right)-\xi^{\frac{2}{3}} \times\left(\frac{x\left(\mathbf{d}_{\mathrm{Q}}\right)}{3}+\operatorname{Tr}\left(\mathbf{x}\left(\mathbf{d}_{\mathrm{Q}}\right) \mathbf{d}_{\mathrm{Q}}\right)\right)$ & $\xi^{2}\left(k\left(\mathbf{d}_{2}\right)-x\left(\mathbf{d}_{2}\right)\right)$ \\
\hline \hline
\end{tabular}

and finally, the exchange matrix is defined as

$$
\tilde{\mathbf{K}}_{\mathrm{S}}=\mathbf{X}(\mathbf{D})+\mathbf{T}_{\mathrm{Q}}^{\mathrm{T}}\left[\mathbf{k}\left(\mathbf{d}_{\mathrm{Q}}\right)-\xi^{\frac{2}{3}} \mathbf{x}\left(\mathbf{d}_{\mathrm{Q}}\right)\right] \mathbf{T}_{\mathrm{Q}}+\Lambda\left(\mathbf{S}-\mathbf{T}_{\mathrm{Q}}^{\mathrm{T}} \mathbf{s} \mathbf{T}_{\mathrm{Q}}\right) .
$$

The various methods are summarized in Table I.

Before proceeding to Sec. III, let us first discuss the scaling of the ADMM variants. Looking at the energy expression in Eqs. (26), (32), and (33), they are composed of one exact exchange term in the small basis and two GGA exchange terms, all of which are clearly linear scaling by standard technologies. ${ }^{11,12}$ The additional two terms with traces of densities and overlaps are naturally linear scaling due to the locality of the basis functions. The evaluation of $\xi$ and $\Lambda$ can easily be made linear scaling by similar arguments. The only term that might present a difficulty is $\mathbf{T}$ due to the presence of the inverse of the small basis overlap matrix, $\mathbf{s}$. This would affect the transformation steps between small and large basis quantities. However, inverse square roots of sparse matrices can be found in a linear scaling fashion, ${ }^{59}$ which might be exploited to obtain linear asymptotic linear scaling.

\section{RESULTS}

\section{A. Computational details}

All calculations have been carried out with a development version of LSDALTON, ${ }^{60}$ compiled with the Intel compiler suite version 14.0.2 in combination with openMPI version 1.6.5. Several basis sets were used, including Pople's split-valence $3-21 G^{61}$ and $6-31 G^{* * 62}$ basis sets, Dunning's correlation-consistent cc-pVDZ and cc-pVTZ basis sets, ${ }^{63}$ and the Karlsruhe $\mathrm{SVP}^{64}$ and TZVPP ${ }^{65}$ basis sets. Density fitting with the def2-QZVPP 66 basis was used for the Coulomb contribution in all cases presented in this paper. Since the same density-fitting basis was used in all calculations, we need only specify the primary and ADMM auxiliary basis sets. Thus, the notation $6-31 \mathrm{G}^{* *} / 3-21 \mathrm{G}$ denotes the use of a $6-31 \mathrm{G}^{* *}$ primary basis set and a $3-21 \mathrm{G}$ auxiliary basis set. All calculations were carried out using the B3LYP functional, ${ }^{67}$ thus including $20 \%$ exact exchange. Whereas the calculations by Guidon et al. ${ }^{4}$ were performed with effective core potentials, the results presented here are for all-electron calculations.

We consider two sets of molecules for benchmarking. The first benchmark set G3* consists of 319 closed shell molecules used for the benchmark of the G3 method by Curtiss et al. ${ }^{68}$ for which all atoms are supported within the basisset combinations used (see below). The second benchmark set M19 is smaller, containing, in addition to 18 molecules previ- ously used by Peach et al. ${ }^{69}$ in a study of excitation energies, benzene, with carbon-carbon and carbon-hydrogen bond distances of 1.395 and $1.0996 \AA$, respectively. ${ }^{30}$

For all ADMM schemes considered here, we have performed calculations with four primary-auxiliary basis combinations (6-31G**/3-21G, cc-pVTZ/3-21G, cc-pVTZ/ccpVDZ, and TZVPP/SVP) and four GGA exchange corrections $\left(\mathrm{B} 88,{ }^{70} \mathrm{PBE},{ }^{71} \mathrm{OPTX}^{72}\right.$ and the exchange part of the KT3 functional, ${ }^{73}$ denoted by B88X, PBEX, OPTX, and KT3X, respectively). The mean errors, maximum absolute errors, and standard deviations for the G3* and M19 test sets are contained in Tables II and III, respectively.

In Sec. III B, we compare the various ADMM models with one another; in Sec. III C, the various GGA functionals are compared. Based on these results, some model functional pairs are singled out for further analysis in Sec. III E. In Sec. III F, we address the question how well a basis set correction calculated using GGA functionals approximates the exact exchange basis set correction using selected models and functionals. In Sec. III G, we compare the ADMM timings with the corresponding LinK timings for the valinomycine and titin molecules, containing 168 and 392 atoms, respectively.

\section{B. Comparison of ADMM models}

Comparing the ADMM statistics for the G3* benchmark set in Table II, the first observation is that ADMMQ performs worse than the other ADMM schemes, with a largest maximum error of $430 \mathrm{~m} E_{\mathrm{h}}$ and a typical error of $10 \mathrm{~m} E_{\mathrm{h}}$. As discussed in Sec. II E, ADMMQ gives an unbalanced treatment of the exact and GGA exchange due to the sensitivity of the SCF procedure to the charge constraint parameter as discussed in Subsection II E. We note that ADMMQ performs reasonably well for some combinations of basis sets and GGA functionals-for example, at the $6-31 \mathrm{G}^{* *} / 3-21 \mathrm{G}$ level of theory. However, being considerably less robust than the other ADMM schemes, ADMMQ will not be considered further here.

Regarding the relative performance of the remaining ADMM models, we first note that ADMM1 performs poorly in the small 3-21G auxiliary basis, with errors as large as 78 $\mathrm{m} E_{\mathrm{h}}$ using 6-31G**/3-21G with OPTX. On the other hand, ADMM1 performs significantly better at the cc-pVTZ/ccpVDZ and TZVPP/SVP levels of theory-in fact, better than all other ADMM models in some cases. Nevertheless, in view of the large ADMM1 errors in the $3-21 \mathrm{G}$ auxiliary basis, we recommend ADMM2 over ADMM1. In fact, the ADMM2 largest absolute errors are half as large as the ADMM1 
TABLE II. Error statistics for the G3* test set. Results are provided in the average error \pm standard deviation (maximum absolute error) format in mEh.

\begin{tabular}{|c|c|c|c|c|c|}
\hline exch. & ADMM1 & ADMM2 & ADMMS & ADMMP & ADMMQ \\
\hline \multicolumn{6}{|c|}{$6-31 \mathrm{G}^{* *} / 3-21 \mathrm{G}$} \\
\hline KT3X & $-2.3 \pm 3.1(22.2)$ & $2.3 \pm 3.0(17.9)$ & $0.0 \pm 2.7(16.2)$ & $-1.2 \pm 3.1(22.7)$ & $11.4 \pm 10.4(69.0)$ \\
\hline OPTX & $-6.4 \pm 4.7(27.4)$ & $-2.8 \pm 3.1(11.6)$ & $-2.3 \pm 3.0(10.0)$ & $-2.9 \pm 3.1(11.9)$ & $8.2 \pm 10.0(62.2)$ \\
\hline PBEX & $-7.3 \pm 5.4(30.0)$ & $-3.9 \pm 4.2(14.5)$ & $-3.2 \pm 4.1(13.5)$ & $-3.8 \pm 4.2(14.7)$ & $7.3 \pm 10.9(62.8)$ \\
\hline B88X & $-7.4 \pm 5.3(31.3)$ & $-4.0 \pm 4.0(15.7)$ & $-3.4 \pm 4.0(14.9)$ & $-4.1 \pm 4.1(16.5)$ & $7.1 \pm 10.7(61.4)$ \\
\hline \multicolumn{6}{|c|}{ cc-pVTZ/3-21G } \\
\hline KT3X & $-6.3 \pm 11.4(71.4)$ & $1.5 \pm 5.7(31.7)$ & $-2.7 \pm 8.5(53.6)$ & $-4.6 \pm 9.6(60.5)$ & $18.5 \pm 14.6(78.1)$ \\
\hline OPTX & $-10.2 \pm 11.8(77.5)$ & $-3.9 \pm 6.3(43.4)$ & $-3.2 \pm 6.4(43.7)$ & $-4.1 \pm 6.7(46.3)$ & $16.8 \pm 16.2(79.9)$ \\
\hline PBEX & $-8.2 \pm 11.2(74.2)$ & $-2.2 \pm 6.4(43.2)$ & $-1.0 \pm 5.8(38.2)$ & $-2.1 \pm 6.3(42.7)$ & $19.2 \pm 17.6(86.1)$ \\
\hline B88X & $-8.35 \pm 11.2(75.2)$ & $-2.3 \pm 6.2(43.7)$ & $-1.3 \pm 5.8(40.0)$ & $-2.5 \pm 6.3(44.6)$ & $18.9 \pm 17.4(83.2)$ \\
\hline \multicolumn{6}{|c|}{ cc-pVTZ/cc-pVDZ } \\
\hline KT3X & $0.6 \pm 1.5(5.8)$ & $1.3 \pm 1.4(6.8)$ & $0.7 \pm 1.5(6.1)$ & $0.4 \pm 1.6(5.7)$ & $3.6 \pm 1.9(10.5)$ \\
\hline OPTX & $0.8 \pm 0.9(3.7)$ & $1.4 \pm 0.9(4.4)$ & $1.5 \pm 0.9(4.6)$ & $1.4 \pm 0.9(4.4)$ & $4.1 \pm 2.1(13.4)$ \\
\hline PBEX & $3.9 \pm 2.7(13.4)$ & $4.5 \pm 2.8(14.2)$ & $4.7 \pm 2.8(14.5)$ & $4.5 \pm 2.8(14.2)$ & $7.4 \pm 3.7(17.6)$ \\
\hline B88X & $3.8 \pm 2.7(13.4)$ & $4.4 \pm 2.8(14.2)$ & $4.6 \pm 2.8(14.4)$ & $4.4 \pm 2.8(14.2)$ & $7.3 \pm 3.7(17.6)$ \\
\hline \multicolumn{6}{|c|}{ TZVPP/SVP } \\
\hline KT3X & $0.5 \pm 3.3(22.1)$ & $1.8 \pm 2.8(11.1)$ & $0.6 \pm 3.5(20.9)$ & $0.1 \pm 3.8(26.7)$ & $8.9 \pm 21.9(337.3)$ \\
\hline OPTX & $-1.6 \pm 2.3(23.6)$ & $-0.5 \pm 1.52(11.0)$ & $-0.3 \pm 1.4(7.5)$ & $-0.6 \pm 1.6(13.8)$ & $8.6 \pm 28.8$ \\
\hline PBEX & $1.0 \pm 2.8(21.7)$ & $2.1 \pm 2.3(10.2)$ & $2.5 \pm 2.2(10.6)$ & $2.1 \pm 2.3(10.4)$ & $11.7 \pm 29.9$ \\
\hline B88X & $0.8 \pm 2.9(22.8)$ & $1.9 \pm 2.4(10.6)$ & $2.2 \pm 2.3(10.5)$ & $1.8 \pm 2.5(12.9)$ & $11.4 \pm 29.4(424.3)$ \\
\hline
\end{tabular}

errors, except at the cc-pVTZ/cc-pVDZ level of theory (where the ADMM1 errors are slightly smaller).

Regarding the performance of the remaining ADMMS and ADMMP models, it is similar or slightly better than that of the ADMM2 model. Over all, the best performance is that of the ADMMS model-in particular, in the calculations with the small 3-21G auxiliary basis. The results for the M19 benchmark set are similar to those for the G3* set, see Table III.

\section{Comparison of GGA functionals}

Comparing the different GGA exchange functionals, we first note that the performances of the PBEX and B88X functionals are similar to each other and often different from those of the KT3X and OPTX functionals. In the $6-31 \mathrm{G}^{* *} / 3-$ $21 \mathrm{G}$ and cc-pVTZ/3-21G calculations, the KT3X functional typically gives the best performance, whereas the OPTX functional gives the best performance for the cc-pVTZ/ccpVDZ and TZVPP/SVP basis-set combinations. Typically,

TABLE III. Error statistics for the M19 test set. Results are provided in the average error \pm standard deviation (maximum absolute error) format in mEh.

\begin{tabular}{|c|c|c|c|c|c|}
\hline exch. & ADMM1 & ADMM2 & ADMMS & ADMMP & ADMMQ \\
\hline \multicolumn{6}{|c|}{$6-31 G^{* *} / 3-21 G$} \\
\hline KT3X & $-4.6 \pm 2.6(9.7)$ & $2.0 \pm 1.6(5.7)$ & $-0.9 \pm 1.0(3.4)$ & $-2.6 \pm 1.7(6.5)$ & $12.8 \pm 7.6(30.1)$ \\
\hline OPTX & $-13.4 \pm 8.1(32.2)$ & $-8.4 \pm 5.3(20.9)$ & $-7.7 \pm 4.8(19.1)$ & $-8.6 \pm 5.3(21.2)$ & $4.6 \pm 2.9(11.9)$ \\
\hline PBEX & $-18.7 \pm 11.7(47.1)$ & $-14.0 \pm 9.1(36.3)$ & $-13.0 \pm 8.6(34.1)$ & $-13.9 \pm 9.1(36.2)$ & $-0.8 \pm 3.5(6.6)$ \\
\hline B88X & $-18.4 \pm 11.4(45.5)$ & $-13.6 \pm 8.7(34.6)$ & $-12.8 \pm 8.3(32.8)$ & $-13.7 \pm 8.8(34.9)$ & $-0.6 \pm 3.0(5.4)$ \\
\hline \multicolumn{6}{|c|}{ cc-pVTZ/3-21G } \\
\hline KT3X & $-2.5 \pm 2.3(9.0)$ & $5.6 \pm 3.9(14.7)$ & $1.5 \pm 2.3(5.8)$ & $-0.7 \pm 2.1(5.9)$ & $20.8 \pm 11.9(47.4)$ \\
\hline OPTX & $-12.2 \pm 7.3(28.6)$ & $-5.9 \pm 3.7(14.7)$ & $-4.8 \pm 3.1(12.4)$ & $-6.1 \pm 3.8(15.1)$ & $12.8 \pm 6.8(26.7)$ \\
\hline PBEX & $-9.8 \pm 6.2(24.4)$ & $-3.6 \pm 2.9(11.0)$ & $-2.3 \pm 2.2(8.1)$ & $-3.6 \pm 2.8(10.8)$ & $15.4 \pm 8.0(31.9)$ \\
\hline B88X & $-9.5 \pm 5.9(23.0)$ & $-3.3 \pm 2.5(9.4)$ & $-2.2 \pm 1.9(7.0)$ & $-3.5 \pm 2.6(9.8)$ & $15.5 \pm 8.1(30.9)$ \\
\hline \multicolumn{6}{|c|}{ cc-pVTZ/cc-pVDZ } \\
\hline KT3X & $1.7 \pm 1.0(3.4)$ & $2.8 \pm 1.5(5.6)$ & $2.0 \pm 1.2(4.1)$ & $1.5 \pm 1.02(3.3)$ & $5.9 \pm 2.9(11.2)$ \\
\hline OPTX & $1.5 \pm 0.8(3.5)$ & $2.4 \pm 1.2(5.4)$ & $2.6 \pm 1.3(5.8)$ & $2.4 \pm 1.2(5.4)$ & $6.1 \pm 3.1(12.2)$ \\
\hline PBEX & $10.6 \pm 6.0(23.4)$ & $11.6 \pm 6.5(25.5)$ & $11.9 \pm 6.6(26.1)$ & $11.7 \pm 6.5(25.5)$ & $15.7 \pm 8.6(34.0)$ \\
\hline B88X & $10.6 \pm 6.0(23.6)$ & $11.6 \pm 6.5(25.7)$ & $11.9 \pm 6.7(26.2)$ & $11.6 \pm 6.5(25.6)$ & $15.6 \pm 8.6(34.2)$ \\
\hline \multicolumn{6}{|c|}{ TZVPP/SVP } \\
\hline KT3X & $5.6 \pm 3.6(12.8)$ & $7.2 \pm 4.3(16.0)$ & $6.0 \pm 3.8(13.4)$ & $5.3 \pm 3.4(12.0)$ & $11.8 \pm 6.5(25.4)$ \\
\hline OPTX & $-2.6 \pm 2.3(8.6)$ & $-1.3 \pm 1.8(6.0)$ & $-1.0 \pm 1.6(5.3)$ & $-1.3 \pm 1.8(6.1)$ & $4.3 \pm 1.9(8.8)$ \\
\hline PBEX & $4.9 \pm 2.5(9.1)$ & $6.2 \pm 3.2(11.9)$ & $6.7 \pm 3.4(12.8)$ & $6.3 \pm 3.2(12.0)$ & $12.3 \pm 6.0(24.3)$ \\
\hline B88X & $4.8 \pm 2.6(9.3)$ & $6.2 \pm 3.2(12.1)$ & $6.5 \pm 3.4(12.8)$ & $6.1 \pm 3.2(12.0)$ & $12.1 \pm 6.1(24.3)$ \\
\hline
\end{tabular}


these functionals perform better than the PBEX functional, originally used for the ADMM1 and ADMM2 calculations. For ADMMS (and ADMMP), the KT3X and OPTX functionals seem especially advantageous. This may be explained by the fact that OPTX has been optimized to reproduce the HF exchange energy accurately, while KT3X consists of the OPTX expression together with some additional terms.

\section{The role of the auxiliary basis}

The ADMM accuracy is expected to improve when making the auxiliary basis set more complete. A natural goal is therefore to achieve a systematic improvement when increasing the size of the auxiliary basis set. Clearly, however, the largest computational gain is achieved using a small auxiliary basis set. From a computational point of view, we would therefore like to keep the auxiliary basis as small as possible without jeopardizing the accuracy of the calculation. One should therefore choose an auxiliary basis with a large overlap with the primary basis. To investigate the role of the auxiliary basis, we first compare the $6-31 \mathrm{G}^{* *} / 3-21 \mathrm{G}$ and cc-pVTZ/3$21 \mathrm{G}$ results, with shared auxiliary and different primary basis sets. For the G3* test set in Table II, the average errors for the smaller $6-31 G^{* *}$ primary basis results are often worse than the larger cc-pVTZ primary basis ones, but there is no clear trend. However, the standard deviations in the $6-31 G^{* *} / 3-21 \mathrm{G}$ case, are significantly smaller than for the cc-pVTZ/3-21G case, as expected. The most accurate results for $\mathrm{G} 3{ }^{*}$ are the ccpVTZ/cc-pVDZ results, in which the auxiliary basis is closest to the primary basis among the four basis set combinations used in this study. However, the results for the M19 test set are less conclusive which makes it hard to make any strong general assessment.

Picking the right auxiliary basis is a question of accuracy vs. efficiency. Based on the results presented in this study, it is not even clear that significantly increasing the auxiliary basis will yield correspondingly improved results. Further investigations are therefore required. An important result is that the cc-pVTZ/3-21G combination seems to be an acceptable

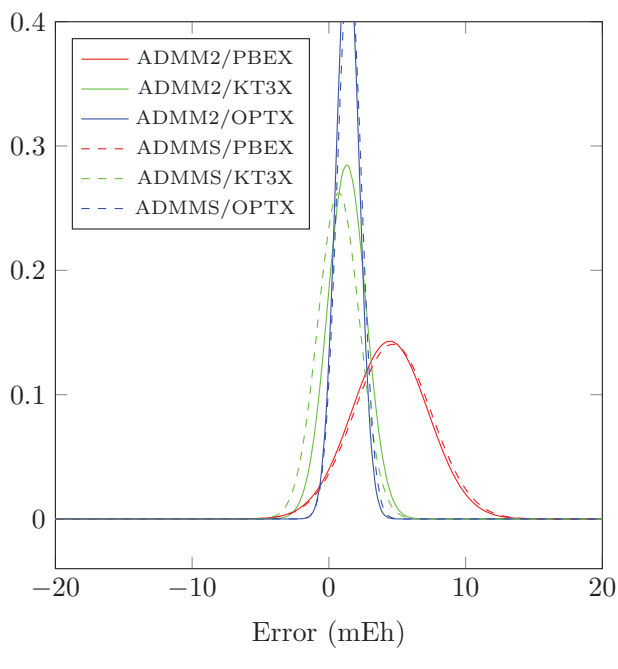

cc-pVTZ/cc-pVDZ

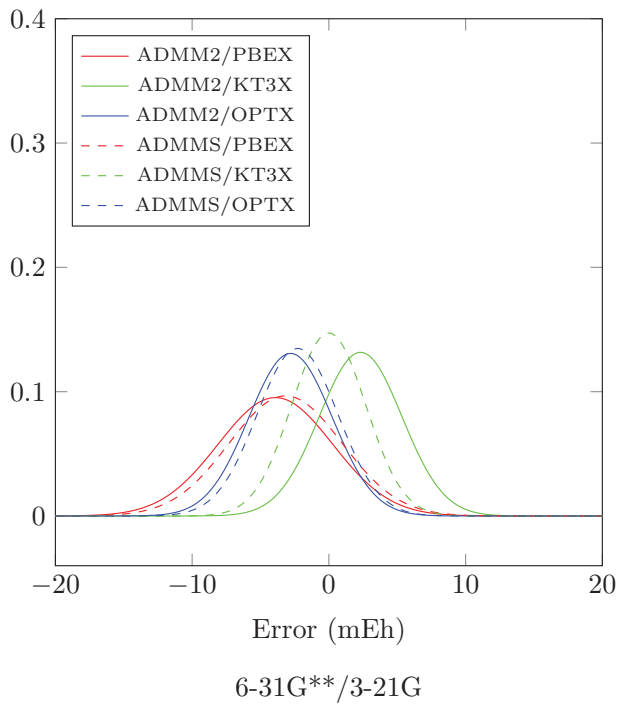

FIG. 1. Chosen error distribution curves for the G3* test set. 
compromise between accuracy and efficiency as will be demonstrated for two large test systems in Subsection III G.

\section{E. Comparison of the ADMM2 and ADMMS models using various functionals}

In Secs. III B and III C, it was concluded that the ADMM2 and ADMMS/ADMMP methods perform best, while among the functionals KT3X and OPTX seem to stand out. For this reason, we choose these models and functionals for a more detailed analysis in the present section, also providing results for the PBEX functional as a reference, since this was originally coupled with the ADMM1 and ADMM2 methods.

In Figures 1 and 2, error distribution curves have been plotted from the various ADMM model and functional combinations. The overall good performance of the ADMMS/KT3X scheme for the 6-31G**/3-21G and cc-pVTZ/3-21G basisset combinations and of the ADMMS/OPTX scheme for the TZVPP/SVP and cc-pVTZ-cc-pVDZ basis-set combinations is well illustrated here.

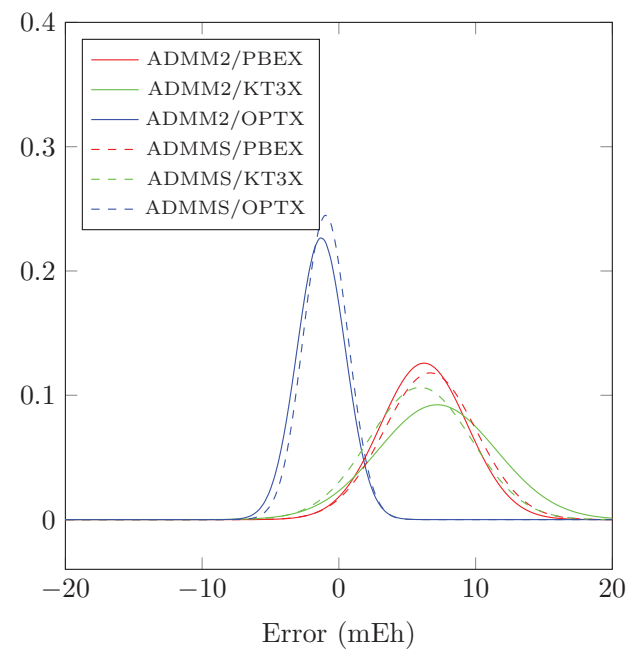

TZVPP/SVP

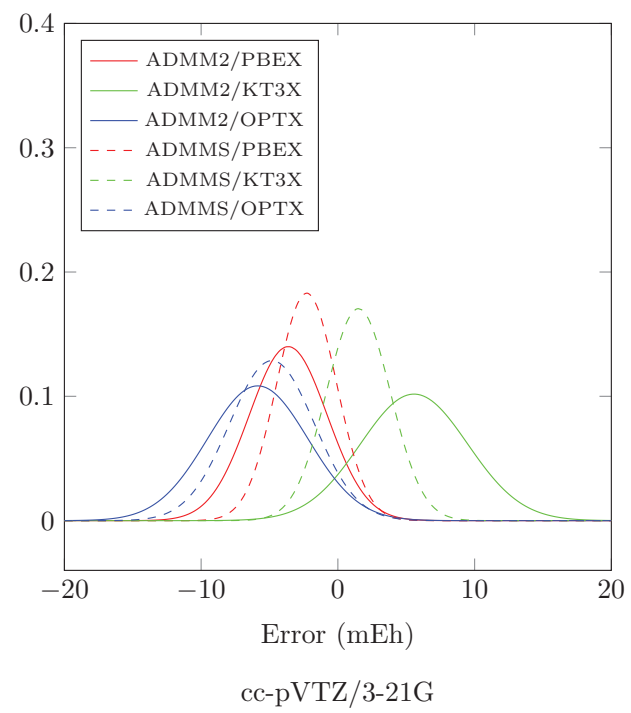

The average error for the ADMMS/KT3X combination is in general closer to zero than is the corresponding ADMM2 error. The standard deviation is often larger for ADMMS/KT3X, although there are some cases of clear improvements here as well, especially for M19. ADMMS with KT3X performs especially well for 6-31G**/3-21G and cc-pVTZ/3$21 \mathrm{G}$, while OPTX has a small average error and standard deviation for TZVPP/SVP. For cc-pVTZ/cc-pVDZ, ADMM2, ADMMP, and ADMMS perform much better for both OPTX and KT3X than for PBEX.

\section{F. Basis set correction for exact and approximate exchange}

One way to interpret Eq. (2) is that the exchange energy in a small basis $(k)$ is corrected with a basis set correction $(X-x)$ evaluated using a DFT exchange functional. It is clearly evident that the accuracy of the ADMM approach depends on how good an approximation is $X-x$ to $K-k$. In Figure 3, these two differences evaluated for the G3* test set are plotted against each other for the ADMM2/PBEX and

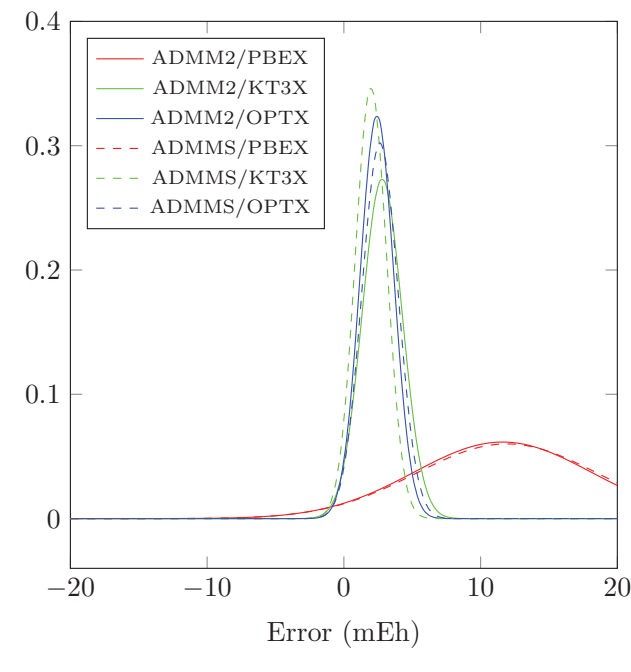

cc-pVTZ/cc-pVDZ

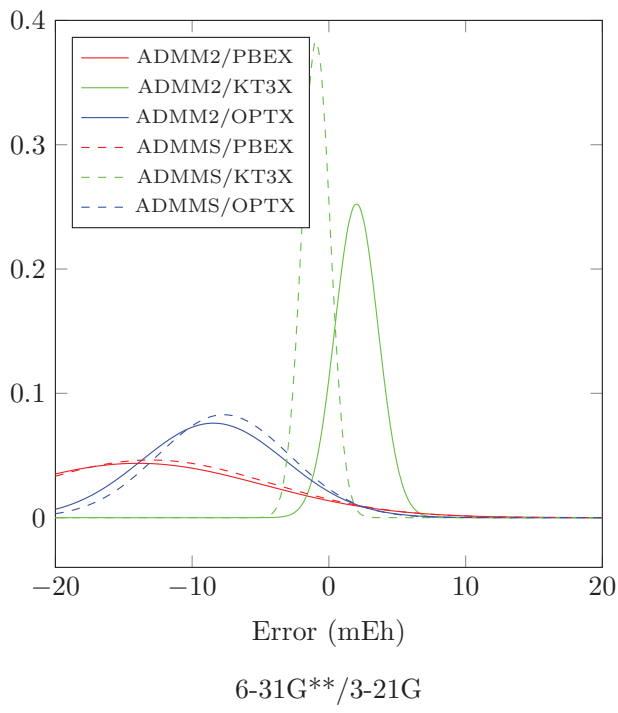

FIG. 2. Chosen error distribution curves for the M19 test set. 


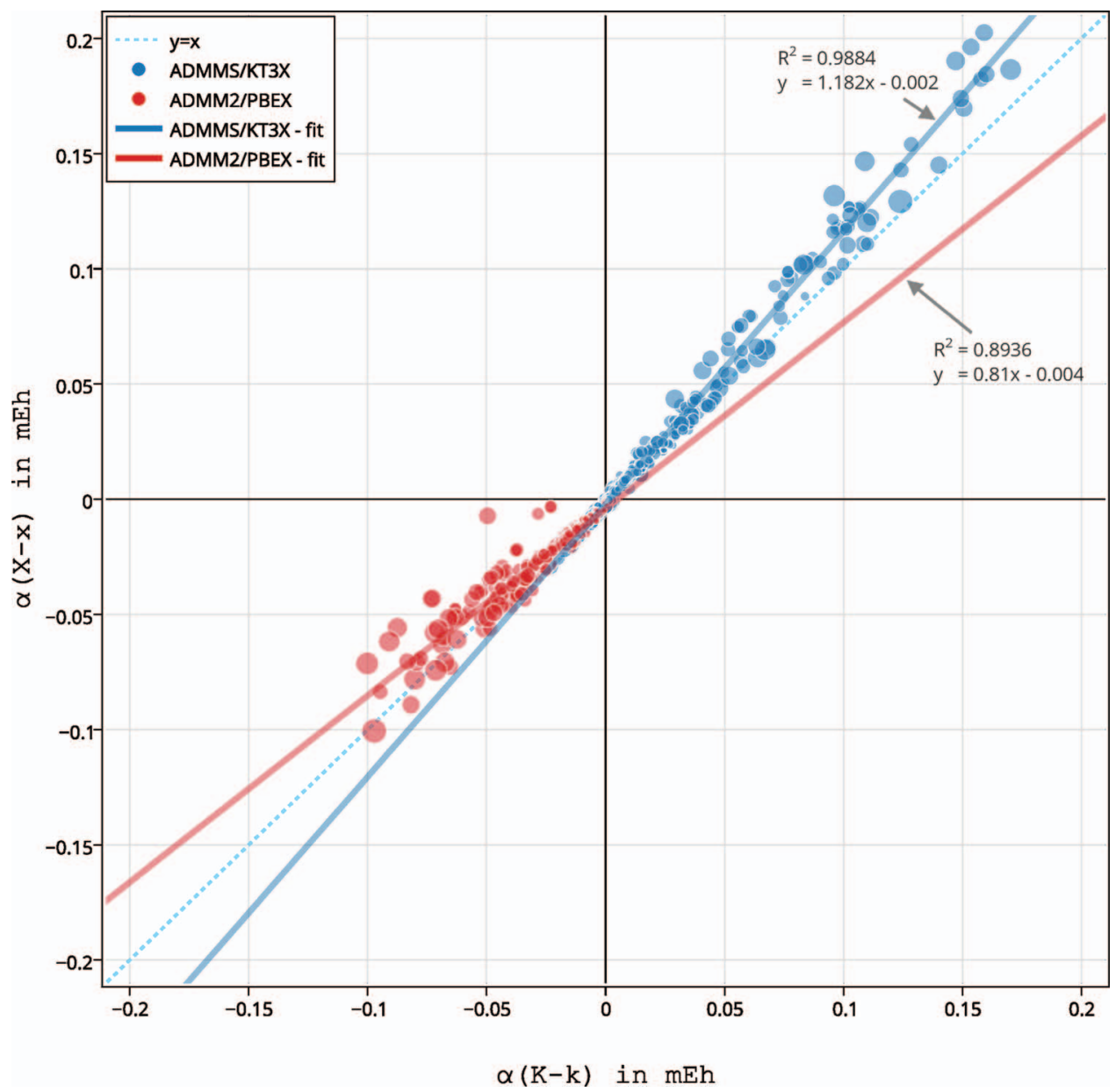

FIG. 3. The exact and approximate exchange basis set corrections for ADMM2/PBEX and ADMMS/KT3X for the G3* test set using the cc-pVTZ/3-21G basis set combination; $\alpha$ is the fraction of exact exchange in B3LYP (i.e., 20\%).

ADMMS/KT3X methods using the cc-pVTZ/3-21G basis set combination.

Ideally, the data points should fall close to the diagonal $y=x$, or at least some linear function. This is satisfied to a large extent by both sets of data points, especially for the ADMMS curve, where $R^{2}$ is much closer to 1 , indicating a better correspondence between the exchange and exact-exchange corrections with ADMMS. The ADMMS curve also gives a somewhat better slope and offset from the origin. The important conclusion for any ADMM type method is that the basic assumption of ADMM, the additivity of the basis set correction is well founded. More specifically, the ADMMS/KT3X combination also improves on the ADMM2/PBEX variant previously published in the literature.

\section{G. Timing results for larger molecules}

Table IV contains some timing and accuracy results for Valinomycin (168 atoms) and Titin (392 atoms) obtained from parallel calculations relative to a LinK exchange reference calculation. With the chosen cc-pVTZ/3-21G basis set combination, this means an $\mathrm{AO}$ basis set of 3600 and 8700 basis functions for Valinomycin and Titin, respectively. The reported timings are for hybrid MPI/OMP calculations with 8 MPI tasks and 16 OMP threads per task. In all the presented calculations, the Coulomb contribution was evaluated using density fitting. For a comparison, the density fitting errors are $-2.3 \mathrm{mE}_{h}$ and $-3.8 \mathrm{mE}_{h}$ for Valinomycin and Titin, respectively. The corresponding exact Coulomb matrix timings are 341.1 and $1895.4 \mathrm{~s}$ per iteration, giving density fitting speedups of factor 54 and 62, respectively.

For both ADMM2/PBEX and ADMMS/KT3X, the calculations converged in the same number of iterations. For ADMMS, the corresponding errors are 8 and -34 mHartree, respectively. For both Valinomycin and Titin, the error caused by ADMM2 is about a factor of four larger than the ADMMS error. In general, the ADMM2 and ADMMS timings are quite similar, except for the ADMM exchange related contribution $\left(t_{K}\right)$, which takes less time for ADMM2 especially in the Titin case. This is due to additional terms related to the evaluation of $\lambda$ and $\Lambda$ only present in ADMMS. The additional cost means however only a negligible increase in the total calculation time for either Valinomycin or Titin, and both ADMM2 and ADMMS remain about a factor of 100 faster for the exchange term relative to the LinK reference. As for total timings, the relative gain of using ADMM is about a factor of 20 for Valinomycin and a factor of 13 for Titin. The reason for the reduced performance gain of ADMM in the latter case is not related to ADMM itself, but rather to the fact that the van Lenthe step $\left(t_{V L}\right)$ becomes the bottle neck for larger systems 
TABLE IV. Timings and accuracy of the ADMM2/PBEX and ADMMS/KT3X methods for valinomycin and titin at the B3LYP/cc-pVTZ/3$21 \mathrm{G}$ level of theory. Listed below are the converged SCF energies $\left(E_{t}\right)$, errors $\Delta E_{t}$ compared to the reference LinK calculation, number of SCF iterations employed $\left(n_{i}\right)$ and timings per iteration for the exchange-matrix construction $\left(t_{K}-\right.$ for ADMM in the small basis), the Coulomb matrix evaluation $\left(t_{J}\right)$, the exchange-correlation functional evaluation $\left(t_{X C}-\right.$ for ADMM also containing $X)$, the van Lenthe optimization step $\left(t_{V L}\right)$. Finally, the total timings are also provided.

\begin{tabular}{lccc}
\hline \hline & Reference & ADMM2 & ADMMS \\
\hline \multicolumn{4}{c}{ Valinomycin } \\
$E_{t}$ (Hartree) & -3793.3264 & -3793.2949 & -3793.3344 \\
$\Delta E_{t}$ (mHartree) & 0.0 & -31.5 & 8.0 \\
$n_{i}$ & 15 & 15 & 15 \\
$t_{K}(\mathrm{~s})$ & 639.3 & 5.9 & 8.8 \\
$t_{J}(\mathrm{~s})$ & 6.3 & 6.3 & 6.3 \\
$t_{X C}(\mathrm{~s})$ & 7.6 & 7.0 & 7.4 \\
$t_{V L}(\mathrm{~s})$ & 5.9 & 6.9 & 7.1 \\
Total $(\mathrm{s})$ & 9962 & 460 & 525 \\
& \multicolumn{4}{c}{ Titin } & & \\
$E_{t}($ Hartree $)$ & -10.97 .8975 & -10787.7834 & -10787.8630 \\
$\Delta E_{t}(\mathrm{mH}$ artree $)$ & 0.0 & -114.1 & -34.4 \\
$n_{i}$ & 15 & 15 & 15 \\
$t_{K}(\mathrm{~s})$ & 2498.8 & 22.8 & 32.1 \\
$t_{J}(\mathrm{~s})$ & 31.3 & 30.7 & 30.3 \\
$t_{X C}(\mathrm{~s})$ & 24.3 & 21.1 & 23.5 \\
$t_{V L}(\mathrm{~s})$ & 80.1 & 83.3 & 76.3 \\
Total (s) & 40436 & 3131 & 3256 \\
\hline \hline
\end{tabular}

due to the cubic scaling Roothaan-Hall diagonalization step. Evaluating the ADMM exchange $\left(t_{K}\right)$ at the cc-pVTZ/3-21G basis requires about the same amount of time as evaluating the exchange-correlation functional $\left(t_{X C}\right)$ and the density fitted Coulomb matrix $\left(t_{J}\right)$.

\section{CONCLUSIONS}

In this paper, several variants of the ADMM exchange method were tested in combination with different GGA exchange-functional corrections and for different basis-set combinations. Other than the already existing ADMM1 and ADMM2 methods, we introduced three new ADMM variants, in which the full orthonormality constraint of the ADMM1 method has been replaced by a simpler scalar constraint. The ADMMQ, ADMMS, and ADMMP variants differ only in formal details while all retain the advantage that only a scalar multiplication of certain terms are required as compared to the unconstrained ADMM2 case. The five methods were tested using two test sets, four basis set combinations, and four exchange functionals for ADMM, yielding the following results:

1. The KT3X and OPTX functionals yield in general better results than either PBEX or B88X.

2. ADMMS (and also ADMMP) yield improved results as compared to ADMM2 and ADMM1.

3. ADMMQ gives the poorest results.

4. For the basis-set combination where the relative gain is the largest, e.g., cc-pVTZ/3-21G, speed-ups factor 19 and 12 for total timings, with corresponding errors of 8 and -35 mHartree at the ADMMS/KT3X level of theory, was observed for Valinomycine and Titin molecules, comprising 168 and 392 atoms, and 3600 and 8700 basis functions, respectively.

5. The underlying assumption that the DFT exchange basis set correction $(X-x)$ is an approximation to the exact exchange energy difference $(K-k)$ is a well founded one.

\section{ACKNOWLEDGMENTS}

This work was supported by the Research Council of Norway (RCN) through CoE Grant No. 179568/V30, by the Norwegian Supercomputing Program (Grant No. NN4654K), and by the European Research Council under the European Union Seventh Framework Program through the Advanced Grant ABACUS, ERC Grant Agreement No. 267683.

${ }^{1}$ J. Kussmann, M. Beer, and C. Ochsenfeld, WIREs: Comput. Mol. Sci. 3,
614 (2013).
${ }^{2}$ R. Izsák, A. Hansen, and F. Neese, Mol. Phys. 110, 2413 (2012).
${ }^{3}$ R. Izsák and F. Neese, Mol. Phys. 111, 1190 (2013).
${ }^{4}$ M. Guidon, J. Hutter, and J. VandeVondele, J. Chem. Theory Comput. 6,
2348 (2010).
${ }^{5}$ I. Panas and J. Almlöf, Int. J. Quantum Chem. 42, 1073 (1992).
6G. R. Ahmadi and J. Almlöf, Chem. Phys. Lett. 246, 364 (1995).
${ }^{7}$ J. Almlöf, D. G. Truhlar, and T. P. Lybrand, Interdiscipl. Sci. Rev. 15, 252
(1990).
${ }^{8}$ I. Panas, J. Almlöf, and M. W. Feyereisen, Int. J. Quantum Chem. 40, 797 (1991).

${ }^{9}$ J. C. Slater, Phys. Rev. 81, 385 (1951).

${ }^{10}$ M. Häser and R. Ahlrichs, J. Comput. Chem. 10, 104 (1989).

${ }^{11}$ E. Schwegler and M. Challacombe, J. Chem. Phys. 105, 2726 (1996).

${ }^{12}$ C. Ochsenfeld, C. A. White, and M. Head-Gordon, J. Chem. Phys. 109, 1663 (1998).

${ }^{13}$ T. Yanai, G. I. Fann, Z. Gan, R. J. Harrison, and G. Beylkin, J. Chem. Phys. 121, 6680 (2004).

${ }^{14}$ R. J. Harrison, G. I. Fann, T. Yanai, Z. Gan, and G. Beylkin, J. Chem. Phys. 121, 11587 (2004).

${ }^{15}$ J. L. Whitten, J. Chem. Phys. 58, 4496 (1973).

${ }^{16}$ J. A. Jafri and J. L. Whitten, J. Chem. Phys. 61, 2116 (1974).

${ }^{17}$ E. J. Baerends, D. E. Ellis, and P. Ros, Chem. Phys. 2, 41 (1973).

${ }^{18}$ B. I. Dunlap, J. W. D. Connolly, and J. R. Sabin, J. Chem. Phys. 71, 3396 (1979).

${ }^{19}$ B. I. Dunlap, J. W. D. Connolly, and J. R. Sabin, J. Chem. Phys. 71, 4993 (1979).

${ }^{20}$ O. Vahtras, J. Almlöf, and M. W. Feyereisen, Chem. Phys. Lett. 213, 514 (1993).

${ }^{21}$ A. M. Köster, J. U. Reveles, and J. M. del Campo, J. Chem. Phys. 121, 3417 (2004).

${ }^{22}$ A. M. Köster, J. M. del Campo, F. Janetzko, and B. Zuniga-Gutierrez, J. Chem. Phys. 130, 114106 (2009).

${ }^{23}$ H. A. Früchtl, R. A. Kendall, R. J. Harrison, and K. G. Dyall, Int. J. Quantum Chem. 64, 63 (1997).

${ }^{24}$ S. Hamel, M. E. Casida, and D. R. Salahub, J. Chem. Phys. 114, 7342 (2001).

${ }^{25}$ F. Weigend, Phys. Chem. Chem. Phys. 4, 4285 (2002).

${ }^{26}$ R. Polly, H.-J. Werner, F. R. Manby, and P. J. Knowles, Mol. Phys. 102, 2311 (2004).

${ }^{27}$ A. Sodt and M. Head-Gordon, J. Chem. Phys. 128, 104106 (2008).

${ }^{28}$ S. Reine, E. Tellgren, A. Krapp, T. Kjærgaard, T. Helgaker, B. Jansík, S. Høst, and P. Sałek, J. Chem. Phys. 129, 104101 (2008).

${ }^{29}$ B. I. Dunlap, J. Mol. Struct. THEOCHEM 501-502, 221 (2000).

${ }^{30}$ P. Merlot, T. Kjærgaard, T. Helgaker, R. Lindh, F. Aquilante, S. Reine, and T. B. Pedersen, J. Comput. Chem. 34, 1486 (2013).

${ }^{31}$ D. Hollman, H. Schaefer, and E. Valeev, J. Chem. Phys. 140, 064109 (2014).

${ }^{32}$ A. Alvarez-Ibarra and A. M. Köster, J. Chem. Phys. 139, 024102 (2013). 
${ }^{33}$ N. H. F. Beebe and J. Linderberg, Int. J. Quantum Chem. 12, 683 (1977).

${ }^{34}$ H. Koch, A. Sánchez de Merás, and T. B. Pedersen, J. Chem. Phys. 118, 9481 (2003).

${ }^{35}$ F. Aquilante, T. B. Pedersen, and R. Lindh, J. Chem. Phys. 126, 194106 (2007).

${ }^{36}$ T. B. Pedersen, F. Aquilante, and R. Lindh, Theor. Chem. Acc. 124, 1 (2009).

${ }^{37}$ F. Aquilante, L. Boman, J. Boström, H. Koch, R. Lindh, A. Sánchez de Merás, and T. B. Pedersen, in Linear-scaling Techniques in Computational Chemistry and Physics: Methods and Applications, Challenges and Advances in Computational Chemistry and Physics Vol. 13, edited by R. Zalesny, M. G. Papadopoulos, P. G. Mezey, and J. Leszczynski (Springer, 2011), Chap. 13, pp. 301-343.

${ }^{38}$ R. A. Friesner, Chem. Phys. Lett. 116, 39 (1985).

${ }^{39}$ R. A. Friesner, J. Chem. Phys. 85, 1462 (1986).

${ }^{40}$ R. A. Friesner, J. Chem. Phys. 86, 3522 (1987).

${ }^{41}$ T. J. Martínez and E. A. Carter, Modern Electronic Structure Theory (World Scientific, Singapore, 1995).

${ }^{42}$ J. C. Light, I. P. Hamilton, and J. V. Lill, J. Chem. Phys. 82, 1400 (1985).

${ }^{43}$ J. C. Light and Z. Bacic, J. Chem. Phys. 85, 4594 (1986).

${ }^{44}$ W. Yang and A. C. Peet, Chem. Phys. Lett. 153, 98 (1988).

${ }^{45}$ A. Yachmenev and W. Klopper, Mol. Phys. 111, 1129 (2013).

${ }^{46}$ F. Neese, F. Wennmohs, A. Hansen, and U. Becker, Chem. Phys. 356, 98 (2009).

${ }^{47}$ R. Izsák and F. Neese, J. Chem. Phys. 135, 144105 (2011).

${ }^{48}$ R. Izsák and F. Neese, J. Chem. Phys. 139, 094111 (2013).

${ }^{49}$ P. Plessow and F. Weigend, J. Comput. Chem. 33, 810 (2012).

${ }^{50}$ E. G. Hohenstein, R. M. Parrish, and T. J. Martínez, J. Chem. Phys. 137, 044103 (2012).

${ }^{51}$ R. M. Parrish, E. G. Hohenstein, T. J. Martínez, and C. D. Sherrill, J. Chem. Phys. 137, 224106 (2012).
${ }^{52}$ E. G. Hohenstein, R. M. Parrish, C. D. Sherrill, and T. J. Martínez, J. Chem. Phys. 137, 221101 (2012).

${ }^{53}$ P.-O. Löwdin, J. Chem. Phys. 18, 365 (1950).

${ }^{54}$ B. C. Carlson and J. M. Keller, Phys. Rev. 105, 102 (1957).

${ }^{55}$ I. Mayer, Int. J. Quantum Chem. 90, 63 (2002).

${ }^{56}$ R. McWeeny, Rev. Mod. Phys. 32, 335 (1960).

${ }^{57}$ A. Borgoo, A. M. Teale, and D. J. Tozer, J. Chem. Phys. 136, 034101 (2012).

${ }^{58}$ A. Borgoo and D. J. Tozer, J. Phys. Chem. A 116, 5497 (2012).

${ }^{59}$ B. Jansík, S. Høst, P. Jørgensen, J. Olsen, and T. Helgaker, J. Chem. Phys. 126, 124104 (2007).

${ }^{60}$ K. Aidas, C. Angeli, K. L. Bak, V. Bakken, R. Bast, L. Boman, O. Christiansen, R. Cimiraglia, S. Coriani, P. Dahle et al., WIREs: Comput. Mol. Sci. 4, 269 (2014)

${ }^{61}$ J. Binkley, J. Pople, and W. Hehre, J. Am. Chem. Soc. 102, 939 (1980).

${ }^{62}$ M. Francl, W. Petro, W. Hehre, J. Binkley, M. Gordon, D. DeFrees, and J. Pople, J. Chem. Phys. 77, 3654 (1982).

${ }^{63}$ T. H. Dunning, Jr., J. Chem. Phys. 90, 1007 (1989).

${ }^{64}$ A. Schäfer, H. Horn, and R. Ahlrichs, J. Chem. Phys. 97, 2571 (1992).

${ }^{65}$ F. Weigend, M. Häser, H. Patzelt, and R. Ahlrichs, Chem. Phys. Lett. 294, 143 (1998).

${ }^{66}$ F. Weigend and R. Ahlrichs, Phys. Chem. Chem. Phys. 7, 3297 (2005).

${ }^{67}$ P. J. Stevens, J. F. Devlin, C. F. Chabalowski, and M. J. Frisch, J. Phys. Chem. 98, 11623 (1994).

${ }^{68}$ L. A. Curtiss, P. C. Redfern, and K. Raghavachari, J. Chem. Phys. 123, 124107 (2005).

${ }^{69}$ M. J. G. Peach, P. Benfield, T. Helgaker, and D. J. Tozer, J. Chem. Phys. 128, 044118 (2008).

${ }^{70}$ A. D. Becke, Phys. Rev. A 38, 3098 (1988).

${ }^{71}$ J. Perdew, K. Burke, and M. Ernzerhof, Phys. Rev. Lett. 77, 3865 (1996).

${ }^{72}$ N. Handy and A. Cohen, Mol. Phys. 99, 403 (2001).

${ }^{73}$ T. Keal and D. Tozer, J. Chem. Phys. 121, 5654 (2004). 